\title{
Approximating Displacement with the Body Velocity Integral
}

\author{
Ross L. Hatton and Howie Choset \\ Carnegie Mellon University \\ \{rlhatton, choset\}@cmu.edu
}

\begin{abstract}
In this paper, we present a technique for approximating the net displacement of a locomoting system over a gait without directly integrating its equations of motion. The approximation is based on a volume integral, which, among other benefits, is more open to optimization by algorithm or inspection than is the full displacement integral. Specifically, we develop the concept of a body velocity integral (BVI), which is computable over a gait as a volume integral via Stokes's theorem. We then demonstrate that, given an appropriate choice of coordinates, the BVI for a gait approximates the displacement of the system over that gait. This consideration of coordinate choice is a new approach to locomotion problems, and provides significantly improved results over past attempts to apply Stokes's theorem to gait analysis.
\end{abstract}

\section{INTRODUCTION}

Locomotion is everywhere. Snakes crawl, fish swim, birds fly, and all manner of creatures walk. The facility with which animals use internal joint motions to move through their environments far exceeds that which has been achieved in artificial systems; consequently there is much interest in raising the locomotion capabilities of such systems to match or surpass those of their biological counterparts. A fundamental aspect of animal locomotion is that it is primarily composed of gaits - cyclic shape motions which efficiently transport the animal. Examples of such gaits include a horse's walking, trotting, and galloping, a fish's translation and turning strokes, and a snake's slithering and sidewinding. The efficacy of these motions, along with the abstraction that they allow from shape to position changes, suggests that gaits will form an equally important part of artificial locomotion.

Here, we are specifically interested in producing tools for designing gaits for mechanical systems which result in desired net position changes. Much prior work in gait design has taken the approach of choosing parameterized basis functions for gaits and simulating the motion of the system while executing the gaits, optimizing the input parameters to find gaits which meet the design requirements. Such optimization with forward simulation is computationally expensive and vulnerable to local minima. Therefore, there is growing interest in using curvature analysis tools, such as Stokes's theorem, to replace the simulation step with a simple volume integration, which is more amenable to optimization. Unfortunately, these Stokes's theorem methods as previously developed are not completely applicable to most interesting systems; either they are restricted to designing small, inefficient motions, or they provide incomplete information about the actual displacement of the system over the course of a gait.

In this paper we address these limitations by developing the concept of a body velocity integral (BVI), which provides an expanded and physically meaningful interpretation of previous locomotion work based on Stokes's theorem. We then identify conditions under which this body velocity integral is a good estimate of the true displacement resulting from a gait. We finish by introducing the notion that rather than being intrinsic to the system, the presence of these conditions is dependent on the choice of parameterization of the system, and demonstrating that this choice of parameterization can be manipulated to ensure their existence.

\section{PRIOR WORK}

Our work builds on the body of locomotion literature which uses geometric mechanics to separate internal shape changes from the external motions they produce. The application of geometric mechanics to locomotion, pioneered by Shapere and Wilczek [1] and further developed by Murray and Sastry [2] and Kelly and Murray [3], provides a powerful mathematical framework for analyzing locomotion. A key product of this work is the development of the reconstruction equation for nonholonomic systems, which relates body velocity to changes in internal shape for a broad class of locomoting systems. We will not rederive the reconstruction equation here; for a thorough treatment, see [4]-[6].

This reconstruction equation has been used in a variety of locomotion contexts. Ostrowski et al. [5], [7] combined the reconstruction equation with Lie bracket theory to generate sinusoidal gaits which translate and rotate a variety of snakelike systems. Bullo and Lynch used the reconstruction equation to decouple the locomotion of kinodynamic systems and design kinematic gaits [8]. More recently, there has been interest in applying these techniques to swimming robots, such as McIsaac and Ostrowski's work on anguilliform (eel-like) robots [9] and Morgansen et al.'s work on fish [10], both of which combine the geometric approach with biomimetic elements. In [11], we introduced the connection vector field as a tool for visualizing the reconstruction equation differentially.

It is not generally possible to integrate the reconstruction equation in closed form, raising difficulties for the inverse problem of finding shape changes which result in desired translations. In special cases, however, Stokes's theorem can be used to find the net motion resulting from gaits [3]. Mukherjee [12] used this principle to analyze the motion 
of rolling disks, and Walsh and Sastry [13] applied it to the case of an isolated three-link robot. Shammas et al. [6], [14] combined this approach with the reconstruction equation to define functions on the shape space of their three-link robots, which allowed the design of gaits resulting in specified rotations. A similar technique was used by Melli et al. [15] and later by Avron and Raz [16] to generate gaits for swimming robots.

\section{BACKGROUND}

The present work makes use of several key techniques borrowed from geometric mechanics, the salient points of which we review here.

\section{A. Three-link Kinematic Snake}

While the principles we are investigating are relevant to a wide range of systems, including fish, differential drive cars, and satellites, in this paper we focus on a particular system, the three-link kinematic snake investigated by Shammas et al. [6], [14]. Illustrated in Fig. 1, this system has a passive wheelset on each link, preventing lateral translation while freely allowing rotation and longitudinal translation. The joint angles $\alpha_{1}$ and $\alpha_{2}$ are actively controlled, and the overall position and orientation of the system are taken as those of the middle link.

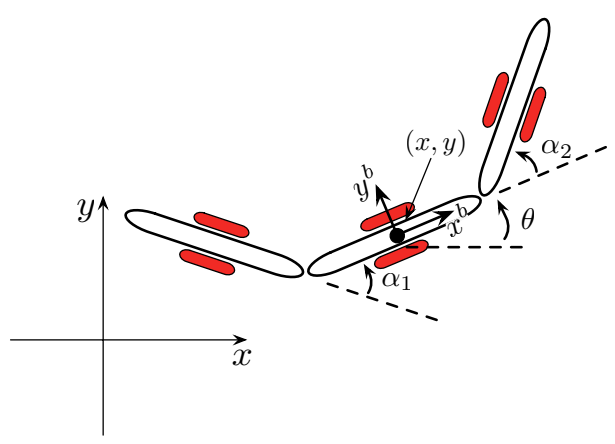

Fig. 1: Model for the three-link kinematic snake. The overall location of the system is the $x, y$ position and orientation $\theta$ of the middle link with respect to the fixed coordinate system. The shape coordinates are $\alpha_{1}$ and $\alpha_{2}$, the angles of the two joints. The passive wheels on each link constrain lateral but not longitudinal or rotational motion.

\section{B. The Reconstruction Equation and the Local Connection}

When analyzing a multi-body locomoting system, it is convenient to separate its configuration space $Q$ into a position space $G$ and a shape space $M$, such that the position $g \in G$ locates the system in the world, and the shape $r \in M$ gives the relative arrangements of its bodies, and then consider how manipulating the shape affects the position. The geometric mechanics community [2]-[6] has addressed this question with the development of the reconstruction equation and the local connection, tools for relating the body velocity of the system, $\xi$, to its shape velocity $\dot{r}$, and accumulated momentum $p$.

The general reconstruction equation is of the form

$$
\xi=-\mathbf{A}(r) \dot{r}+\boldsymbol{\Gamma}(r) p,
$$

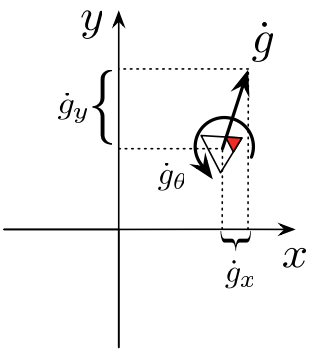

(a) World velocity.

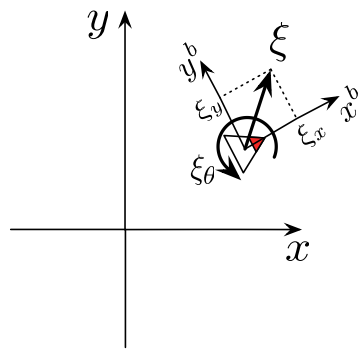

(b) Body velocity.
Fig. 2: Two representations of the velocity of a robot. The robot, represented by the triangle, is translating up and to the right, while spinning counterclockwise. In (a), the world velocity, $\dot{g}$, is measured with respect to the global frame. The body velocity, $\xi$, in (b) is the world velocity represented in the robot's instantaneous local coordinate frame.

where $\xi$ is the body velocity of the system, $\mathbf{A}(r)$ is the local connection, a matrix which relates joint to body velocity, $\boldsymbol{\Gamma}(r)$ is the momentum distribution function, and $p$ is the generalized nonholonomic momentum, which captures how much the system is "coasting" at any given time [4]. The body velocity, illustrated for a planar system in Fig. 2(b), is the position velocity as expressed in the instantaneous local coordinate frame of the system. For systems which translate and rotate in the plane, i.e. which have an $S E(2)$ position space, the body and world velocities are related to each other as

$$
\xi=\left[\begin{array}{ccc}
\cos \theta & \sin \theta & 0 \\
-\sin \theta & \cos \theta & 0 \\
0 & 0 & 1
\end{array}\right] \dot{g}
$$

where $\theta$ is the system's orientation.

For systems which are sufficiently constrained, the generalized momentum drops out, and the system behavior is dictated by the kinematic reconstruction equation,

$$
\xi=-\mathbf{A}(r) \dot{r}
$$

in which the local connection thus acts as a kind of Jacobian, mapping from velocities in the shape space to the corresponding body velocity. For the rest of this paper, we will limit our attention to these kinematic systems.

Kinematic Snake Example 1: For the kinematic snake, we take the position as $g=(x, y, \theta) \in S E(2)$ of the middle link and the shape as the joint angles $r=\left[\begin{array}{ll}\alpha_{1} & \alpha_{2}\end{array}\right]^{T} \in \mathbb{R}^{2}$, as in Fig 1. Outside of singularities, the passive wheelsets constitute three independent constraints, equal in number to the position coordinates, so the system has no direction in which to coast and behaves kinematically. As detailed in [6], the constraints define the local connection for the system, such that the reconstruction equation, normalized for link length, is

$$
\xi=\left[\begin{array}{l}
\xi_{x} \\
\xi_{y} \\
\xi_{\theta}
\end{array}\right]=-\frac{1}{D}\left[\begin{array}{cc}
1+\cos \left(\alpha_{2}\right) & 1+\cos \left(\alpha_{1}\right) \\
0 & 0 \\
-\sin \left(\alpha_{2}\right) & -\sin \left(\alpha_{1}\right)
\end{array}\right]\left[\begin{array}{c}
\dot{\alpha}_{1} \\
\dot{\alpha}_{2}
\end{array}\right],
$$




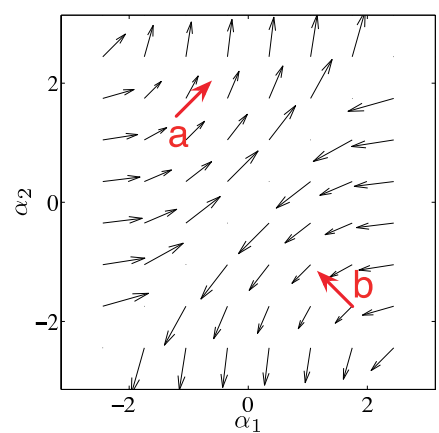

(a) $\overrightarrow{\mathbf{A}} \xi_{x}$

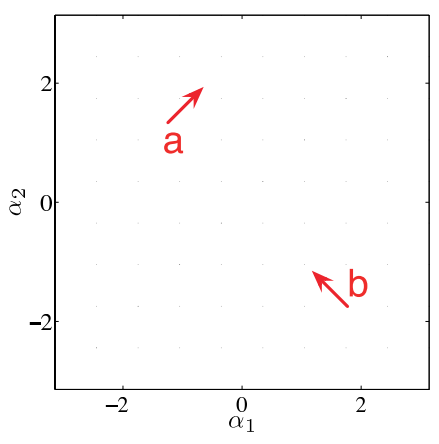

(b) $\overrightarrow{\mathbf{A}} \xi_{y}$

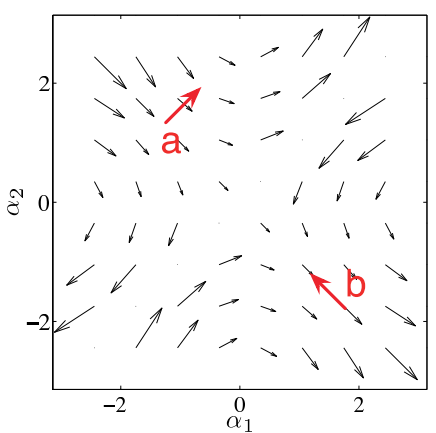

(c) $\overrightarrow{\mathbf{A}} \xi_{\theta}$

Fig. 3: The connection vector fields for the kinematic snake. Due to singularities in the vector fields at the lines $\alpha_{1}= \pm \pi, \alpha_{2}= \pm \pi$, and $\alpha_{1}=\alpha_{2}$, the magnitudes of the vector fields have been scaled to their arctangents for readability. The $\dot{r}$ vectors at a and $\mathbf{b}$ will produce pure forward translation and pure negative rotation, respectively.

\section{Connection vector fields}

Each row of the local connection $\mathbf{A}(r)$ can be considered as defining a vector field on the shape space whose dot product with the shape velocity produces the corresponding component of the body velocity,

$$
\xi_{i}=\overrightarrow{\mathbf{A}}^{\xi_{i}}(r) \cdot \dot{r}
$$

where, for convenience, we wrap the negative sign into the vector field definition.

Considering the local connection as a set of vector fields with the dot product operator provides strong geometric intuition for understanding the relationship between shape and position motions. The geometric interpretation of the dot product in (5) is

$$
\xi_{i}=\overrightarrow{\mathbf{A}}^{\xi_{i}}(r) \cdot \dot{r}=\left\|\overrightarrow{\mathbf{A}}^{\xi_{i}}(r)\right\|\|\dot{r}\| \cos \Theta,
$$

where $\Theta$ is the angle between the vectors. Taking the $\cos \Theta$ term as a measure of the alignment of $\overrightarrow{\mathbf{A}}^{\xi_{i}}(r)$ and $\dot{r}, \xi_{i}$ is positive, negative, or zero when the two vectors have correspondingly positive, negative, or zero alignment, and is scaled by the magnitudes of $\overrightarrow{\mathbf{A}}^{\xi_{i}}$ and $\dot{r}$.

Kinematic Snake Example 2: The connection vector fields for the kinematic snake with reconstruction equation as in (4) are shown in Fig. 3 along with two example $\dot{r}$ vectors. At position $\mathbf{a}, \dot{r}$ is aligned with $\overrightarrow{\mathbf{A}}^{\xi_{x}}$ and orthogonal to $\overrightarrow{\mathbf{A}}^{\xi_{\theta}}$, and will thus produce forward (positive longitudinal) motion with no rotation. At $\mathbf{b}, \dot{r}$ is orthogonal to $\overrightarrow{\mathbf{A}}^{\xi_{x}}$ and anti-aligned with $\overrightarrow{\mathbf{A}}^{\xi_{\theta}}$, and will thus produce negative rotation with no translation.

\section{Shape Changes}

To describe operations in the shape space of a robot, we define shape changes, gaits, and image-families of gaits.

Definition 3.1 (Shape change): A shape change $\psi \in \Psi$ is a trajectory in the shape space $M$ of the robot over an interval $[0, T]$, i.e., the set of all shape changes is

$$
\Psi=\left\{\psi \in C^{1} \mid \psi:[0, T] \rightarrow M\right\}
$$

where $\psi(0), \psi(T) \in M$ are respectively the start and end shapes.

Definition 3.2 (Gait): A gait $\phi \in \Phi$ is a cyclic shape change, i.e.

$$
\Phi=\{\phi \in \Psi \mid \phi(0)=\phi(T)\} .
$$

Note that a gait has a defined start shape $\phi(0)$; two gaits whose images in $M$ are the same closed curve, but with different start points, are distinct.

Definition 3.3 (Image-family): The image-family of a gait is the set of all gaits which share its image (i.e., trace out the same closed curve) in $M$.

\section{E. Stokes's Theorem}

In general, to calculate the displacement resulting from a gait, we must use the local connection to find the body velocity and then integrate this velocity over time. In some cases, however, we can use Stokes's theorem to replace this time integral with an area integral. By this theorem, the line integral along a closed curve on a vector field is equal to the integral of the curl of that vector field over a surface bounded by the curve. For example, for systems with two shape variables, the integral of a component of the body velocity over a gait is thus

$$
\begin{aligned}
\int_{0}^{T} \xi_{i}(\tau) d \tau & =\int_{0}^{T} \overrightarrow{\mathbf{A}}^{\xi_{i}}(\phi(\tau)) \cdot \dot{\phi}(\tau) d \tau \\
& =\int_{\phi} \overrightarrow{\mathbf{A}}^{\xi_{i}}(r) d r \\
& =\iint_{\phi_{a}} \operatorname{curl} \overrightarrow{\mathbf{A}}^{\xi_{i}}(r) d r
\end{aligned}
$$

where $\phi_{a}$ is the area on $M$ enclosed by the gait. If a component of the world velocity is always equal to a component of the body velocity, i.e., $\dot{g}_{j}=\xi_{j}$ for a given $j$, then we can apply Stokes's theorem to find the net displacement in that direction over the course of a gait, by identifying

$$
\Delta g_{j}=\int_{0}^{T} \dot{g}_{j}(\tau) d \tau=\int_{0}^{T} \xi_{j}(\tau) d \tau
$$


and substituting into (9) through (11).

In addition to evaluating the displacement over gaits, (11) offers a powerful means of addressing the inverse problem of designing gaits. By plotting the curl of the connection vector field as a height function $\mathbf{H}^{\zeta_{j}}(r)$ on the shape space, we can easily identify gaits which produce various displacements by inspection. Rules of thumb for designing curves which produce desired values of the integral in (11) are given in [6]:

1) Non-zero integral (I) A loop in a region where the sign of $\mathbf{H}^{\zeta_{j}}(r)$ is constant will produce a non-zero integral. The sign of the integral will depend on the sign of $\mathbf{H}^{\zeta_{j}}(r)$ and the orientation of the loop (the direction in which it is followed).

2) Non-zero integral (II) A figure-eight across a line $\mathbf{H}^{\zeta_{j}}(r)=0$, where each half is in a region where the sign of $\mathbf{H}^{\zeta_{j}}(r)$ is constant, will produce a non-zero integral.

3) Zero integral A loop that encloses equally positive and negative regions of $\mathbf{H}^{\zeta_{j}}(r)$, such that the integrals over the positive and negative regions cancel each other out, will produce a zero integral.

Kinematic Snake Example 3: From (2), we see that $\xi_{\theta}=\dot{g}_{\theta}$ for systems with an $S E(2)$ position space, as is the case for the kinematic snake. For these systems we can thus use Stokes's theorem to design gaits which produce desired net rotations of these systems. The rotational height function for the kinematic snake,

$$
\mathbf{H}^{\zeta_{\theta}}=\frac{\partial \overrightarrow{\mathbf{A}}_{2}^{\xi_{\theta}}}{\partial \alpha_{1}}-\frac{\partial \overrightarrow{\mathbf{A}}_{1}^{\xi_{\theta}}}{\partial \alpha_{2}}
$$

is plotted in Fig. 4 along with two gait image-families. In Fig. 4(a), the loop encircles an area over which the integral $\iint_{\phi_{a}} \mathbf{H}^{\zeta_{\theta}} d \alpha_{1} d \alpha_{2}$ is zero, so the net rotation resulting from any gait in that image-family will be zero. In Fig. 4(b), each half of the figure-eight pattern is on a different side of the $\mathbf{H}^{\zeta_{\theta}}=0$ line, and the two halves have opposite orientations. The integrals over the two areas will thus sum together, and the snake will gain a net rotation from any gait in the family. The sign of this net rotation will depend on the orientation of the curve (the direction in which the curve is followed) [6], [15].

\section{The Body Velocity INTEGRAL}

The convenience and simplicity of using the $\mathbf{H}^{\zeta_{\theta}}$ height function to design gaits resulting in desired rotations makes it tempting to apply the same techniques to designing gaits which produce specified rotations. Unfortunately, this is not generally possible, as the integral of body velocity provided by the translational height functions does not correspond to the resulting displacement. To see why this is the case, consider that there are two senses in which "integrating the body velocity over time" can be interpreted. In the first, most natural sense, we integrate to find the resulting displacement. This

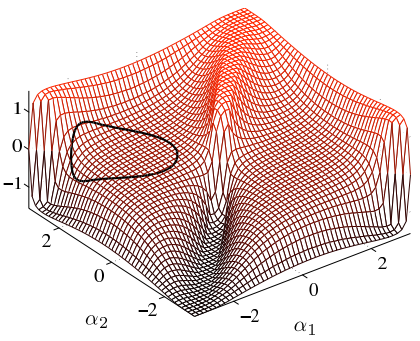

(a)

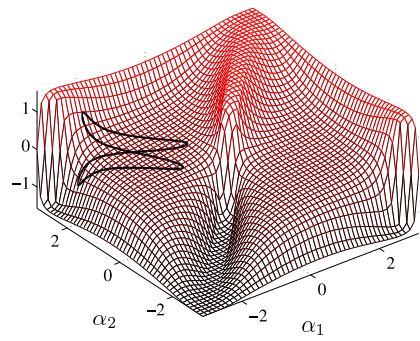

(b)
Fig. 4: The connection height function for rotation, $\mathbf{H}^{\zeta_{\theta}}$ for the kinematic snake, along with two gait image-families. In (a), the loop encircles equal positive and negative areas of the height function, so any gait from this family will result in zero net rotation. The two loops of the image-family in (b) have opposite orientations and encircle oppositely-signed regions of $\mathbf{H}^{\zeta_{\theta}}$, so gaits from this family will result in a non-zero net rotation. To accommodate the singularity along the $\alpha_{1}=\alpha_{2}$ line, the height function is scaled to its arctangent for display.

quantity is found by the nonlinear iterative integral

$g(t)=\left[\begin{array}{l}x(t) \\ y(t) \\ \theta(t)\end{array}\right]=\int_{0}^{t}\left[\begin{array}{ccc}\cos \theta(\tau) & -\sin \theta(\tau) & 0 \\ \sin \theta(\tau) & \cos \theta(\tau) & 0 \\ 0 & 0 & 1\end{array}\right]\left[\begin{array}{l}\xi_{x}(\tau) \\ \xi_{y}(\tau) \\ \xi_{\theta}(\tau)\end{array}\right] d \tau$,

in which the $x$ and $y$ components of the body velocity are rotated into the world frame at each time.

The second sense of integrating $\xi$ is to take its simple vector integral $\zeta$,

$$
\zeta(t)=\left[\begin{array}{l}
\zeta_{x}(t) \\
\zeta_{y}(t) \\
\zeta_{\theta}(t)
\end{array}\right]=\int_{0}^{t}\left[\begin{array}{l}
\xi_{x}(\tau) \\
\xi_{y}(\tau) \\
\xi_{\theta}(\tau)
\end{array}\right] d \tau
$$

where we term $\zeta$ the body velocity integral, or BVI. Physically, the BVI corresponds to the raw odometry for the robot, i.e., the net forwards minus backwards motion in each body direction, and it does not account for changes in the alignment between body and world directions, i.e. the relative orientation of the body and world frames.

The area integrals under the height functions correspond to this second sense of integration, and as such cannot generally be used to determine the displacement resulting from a given gait. For instance, consider how the kinematic snake moves while executing gaits from the gait image family depicted in Fig. 5. On the height functions, the gait family encircles a positive area on $\mathbf{H}^{\zeta_{x}}$, a zero area on $\mathbf{H}^{\zeta_{y}}$, and a net-zero area on $\mathbf{H}^{\zeta_{\theta}}$. As in the example from Section III-E, we can conclude from the net zero integral on $\mathbf{H}^{\zeta_{\theta}}$ that the threelink robot will undergo no net rotation over the course of any gait from this family. However, it will undergo intermediate rotation, and, by comparing the translational BVI $\left(\zeta_{x}, \zeta_{y}\right)$ against the actual $(x, y)$ displacement resulting from gaits in this family, as in Fig. 6, we see that the BVI does not equal the displacement. Further, as the BVI has a single value for all gaits in an image family, but the displacement is dependent on the choice of start/end shape, it is apparent that we can have no expectation of finding a map from BVI to displacement.

There is a bright side, however. While our example shows that we cannot always take the BVI as an indication of the 

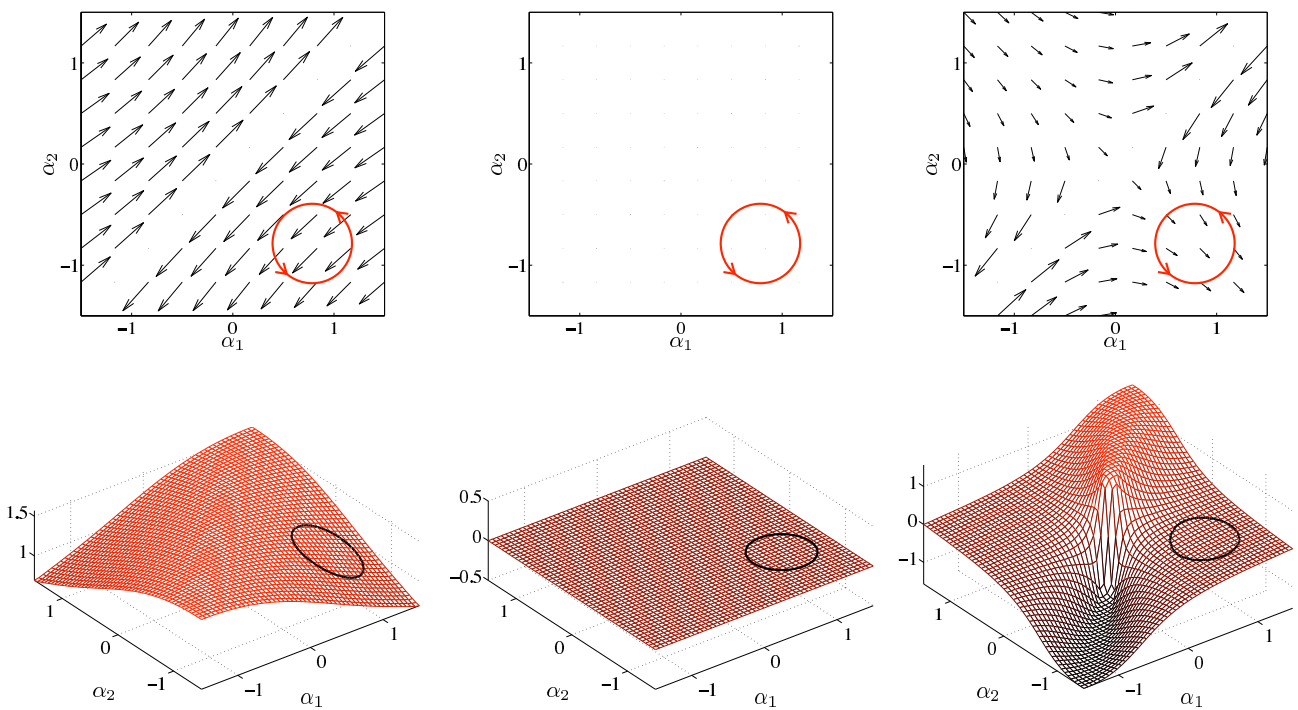

$x$

$y$

$\theta$

Fig. 5: Example gait image-family overlaid on the connection vector fields and height functions of the kinematic snake.

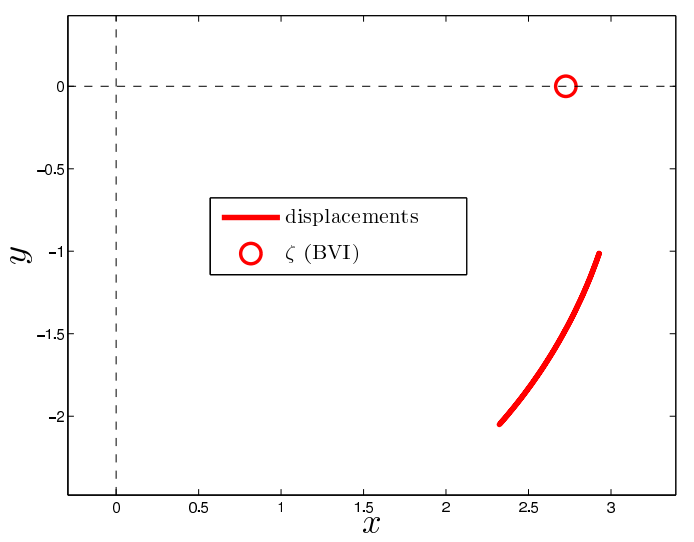

Fig. 6: The BVI and locus of displacements corresponding to the image family of gaits for the kinematic snake robot depicted in Fig. 5 are represented respectively by the circle on the $x$-axis and the arc-section. The BVI only depends on an area integral over the area enclosed by the gait and is thus the same for all gaits in the family. The displacement, however, also depends on the start/end shape of the gait, and there is thus a range of displacements, each corresponding to one starting point in the image family. The displacements are on the order of one link length of the robot.

net displacement, it does not show that we can never do so. To investigate the existence of gaits for which the BVI is a reliable measure of displacement, we consider the error $\varepsilon_{\zeta}$ between the BVI and displacement over an arbitrary gait. By subtracting (14) from (15) and combining the integrals, we can express this error as

$$
\varepsilon_{\zeta}=\zeta-g=\int_{0}^{t}\left[\begin{array}{ccc}
1-\cos \theta & \sin \theta & 0 \\
-\sin \theta & 1-\cos \theta & 0 \\
0 & 0 & 0
\end{array}\right]\left[\begin{array}{l}
\xi_{x} \\
\xi_{y} \\
\xi_{\theta}
\end{array}\right] d \tau
$$

with an implicit dependence on $\tau$ for all variables inside the integral. From (16), we can easily identify a condition which will guarantee that the error term is small: If $\theta(t)$ remains small for all $t$, then the matrix in the integrand will remain close to a zero matrix for all $t$, and the error $\varepsilon_{\zeta}$ will thus be close to a zero vector. We will thus turn our attention to finding conditions for which this small angle condition holds true.

Trivially, small gaits offer $\theta$ no opportunity to grow large; these gaits have been studied extensively in the controls literature with the aid of Lie algebra analysis [2], [5], [10], [15]. These gaits, however, spend more energy in high-frequency oscillations than they do in producing net motion, and are thus inefficient; we are interested in designing larger, more efficient gaits. To this end, we observe that as the orientation of the system over a gait is

$$
\theta(t)=\int_{0}^{t} \xi_{\theta}(\tau) d \tau=\int_{\phi(0)}^{\phi(t)} \overrightarrow{\mathbf{A}}^{\xi_{\theta}}(r) d r
$$

its value at any time is bounded to

$$
|\theta(t)| \leq \int_{\phi(0)}^{\phi(t)}\left|\overrightarrow{\mathbf{A}}^{\xi_{\theta}}(r) d r\right|
$$

For gaits in regions for which $\left\|\overrightarrow{\mathbf{A}}^{\xi_{\theta}}(r)\right\|$ vanishes, $\theta(t)$ and $\varepsilon_{\zeta}(t)$ are thus guaranteed to be small, and the BVIs of those gaits are thus good approximations of their displacements.

The $\overrightarrow{\mathbf{A}}^{\xi_{\theta}}(r)$ vector field for the kinematic snake in Fig. 5 clearly does not become small at any point, so there are no regions over which we can use the BVI to design gaits. However, we will see below that the connection vector fields depend on the representation of the system, and that there are sets of connection vector fields for the kinematic snake in which $\overrightarrow{\mathbf{A}}^{\xi_{\theta}}(r)$ does vanish over a region of $M$. 


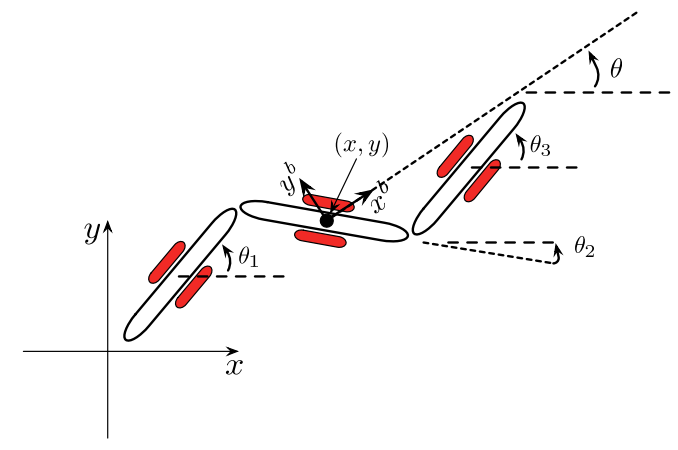

Fig. 7: Orientation of the kinematic snake with the new choice of coordinates The orientation $\theta$ is the mean of the individual link orientations $\theta_{1}, \theta_{2}$, and $\theta_{3}$. Note that the body frame directions $x^{b}$ and $y^{b}$ are respectively aligned with and perpendicular to the orientation line, and not to the central axis of the middle link.

\section{The BVI in A New Body Frame}

We now make a major departure from previous approaches, and consider the effect of changing the parameterization of the problem. For instance, up to this point in our analysis of the kinematic snake we have been considering $g$ as the position and orientation of its middle link, as shown in Fig. 1. This choice of coordinates is customary in the treatment of threelink systems [3], [6], [11], [15], [16], and has the advantage of providing for the simple representation of the system constraints in the body frame. An equally valid choice of coordinates, however, would be to take $\theta$ not as the orientation of the middle link, but as the mean orientation of all three links, i.e.,

$$
\theta=\frac{\theta_{1}+\theta_{2}+\theta_{3}}{3},
$$

where $\theta_{i}$ is the orientation of the $i$ th link, as in Fig. 7 .

This choice of coordinates has two key benefits over the previous choice. First, the orientation $\theta$ matches an intuitive understanding of the "orientation" of the system much more strongly with the new choice than for the old choice, as illustrated in Fig. 8. Second, and more importantly, the $\overrightarrow{\mathbf{A}}^{\xi_{\theta}}$ connection vector field vanishes in key regions, allowing the use of the height functions to design gaits with specific displacements.

To gain insight into this second benefit, consider that the orientations $\theta_{1}$ and $\theta_{3}$ of the outer links of the kinematic snake in Fig. 7 can be expressed in terms of the orientation $\theta_{2}$ of the middle link and the joint angles $\alpha_{1}$ and $\alpha_{2}$ from Fig. 1 as

$$
\begin{aligned}
& \theta_{1}=\theta_{2}-\alpha_{1} \\
& \theta_{3}=\theta_{2}+\alpha_{2} .
\end{aligned}
$$

Consequently, $\theta_{\text {new }}$, the mean orientation of the three links in (19), is thus

$$
\begin{aligned}
\theta_{\text {new }} & =\frac{\left(\theta_{2}-\alpha_{1}\right)+\theta_{2}+\left(\theta_{2}+\alpha_{1}\right)}{3} \\
& =\theta_{2}+\frac{\left(-\alpha_{1}\right)+\alpha_{2}}{3}
\end{aligned}
$$

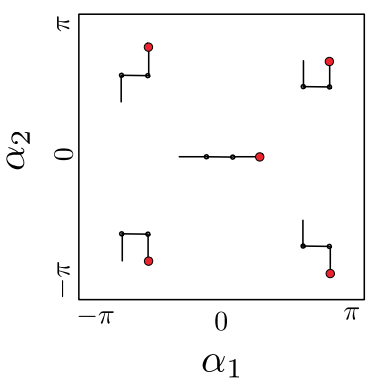

(a) Old coordinate choice

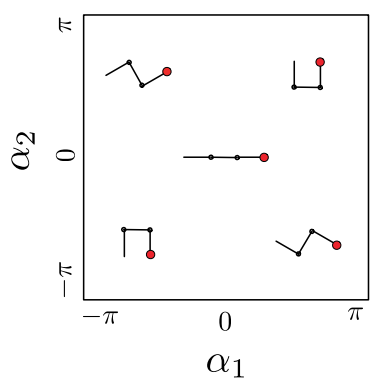

(b) New coordinate choice
Fig. 8: A three-link system in various configurations. In both (a) and (b), the system is shown at five points on the shape space with reference orientation $\theta=0$. In (a), $\theta$ is the orientation of the middle link, while in (b), $\theta$ is the mean orientation of the links. The system is much more consistently "pointing to the right" (an intuitive interpretation of $\theta=0$ ) in (b) than in (a).

and the rotational velocity $\dot{\theta}_{\text {new }}$ of this mean orientation line is then

$$
\dot{\theta}_{\text {new }}=\dot{\theta}_{2}+\frac{\left(-\dot{\alpha}_{1}\right)+\dot{\alpha}_{2}}{3} .
$$

Given that $\theta_{2}$ and $\dot{\theta}_{2}$ are respectively the orientation and angular velocity of the old body frame, $\theta_{\text {old }}$ and $\dot{\theta}_{\text {old }}$, the new angular velocity is

$$
\begin{aligned}
\dot{\theta}_{\text {new }} & =\dot{\theta}_{\text {old }}+\frac{\left(-\dot{\alpha}_{1}\right)+\dot{\alpha}_{2}}{3} \\
& =\overrightarrow{\mathbf{A}}_{\text {old }}^{\xi_{\theta}} \cdot \dot{r}+\left[\begin{array}{ll}
-\frac{1}{3} & \frac{1}{3}
\end{array}\right] \cdot \dot{r} \\
& =\left(\overrightarrow{\mathbf{A}}_{\text {old }}^{\xi_{\theta}}+\left[\begin{array}{ll}
-\frac{1}{3} & \frac{1}{3}
\end{array}\right]\right) \cdot \dot{r} .
\end{aligned}
$$

As the new rotational connection vector field, $\overrightarrow{\mathbf{A}}_{\text {new }}^{\xi_{\theta}}$, relates the new angular velocity to the shape velocity by

$$
\dot{\theta}_{\text {new }}=\overrightarrow{\mathbf{A}}_{\text {new }}^{\xi_{\theta}} \cdot \dot{r},
$$

we see from (27) that it is of the form

$$
\overrightarrow{\mathbf{A}}_{\text {new }}^{\xi_{\theta}}=\overrightarrow{\mathbf{A}}_{\text {old }}^{\xi_{\theta}}+\overrightarrow{\mathbf{B}}^{\xi_{\theta}},
$$

with $\overrightarrow{\mathbf{B}}^{\xi_{\theta}}=\left[\begin{array}{ll}-\frac{1}{3} & \frac{1}{3}\end{array}\right]$. Representing this sum graphically, as in Fig. 9, we see the effect of the change of coordinates on the local connection: The connection modifier $\overrightarrow{\mathbf{B}}^{\xi_{\theta}}$ is approximately equal to the negative of the old rotational connection vector field $\overrightarrow{\mathrm{A}}_{\text {old }}^{\xi_{\theta}}$ in the circled regions, and thus nullifies it when they are summed together.

By a similar derivation, the (row vector) translational components of of the new local connection are related to those of the old local connection by the rotation

$$
\left[\begin{array}{c}
\overrightarrow{\mathbf{A}}_{\text {new }}^{\xi_{x}} \\
\overrightarrow{\mathbf{A}}_{\text {new }}^{\xi_{y}}
\end{array}\right]=\left[\begin{array}{rr}
\cos \beta & \sin \beta \\
-\sin \beta & \cos \beta
\end{array}\right]\left[\begin{array}{c}
\overrightarrow{\mathbf{A}}_{\text {old }}^{\xi_{x}} \\
\overrightarrow{\mathbf{A}}_{\text {old }}^{\xi_{y}}
\end{array}\right],
$$

where

$$
\beta=\theta_{\text {new }}-\theta_{\text {old }}=\frac{\left(-\dot{\alpha}_{1}\right)+\dot{\alpha}_{2}}{3} .
$$

The connection vector fields and height functions corresponding to the new coordinate choice are shown in Fig. 10, along with the same gait image-family as in Fig. 5. As the gait image-family shown is in the null region of $\overrightarrow{\mathbf{A}}_{\text {new }}^{\xi_{\theta}}$, the 


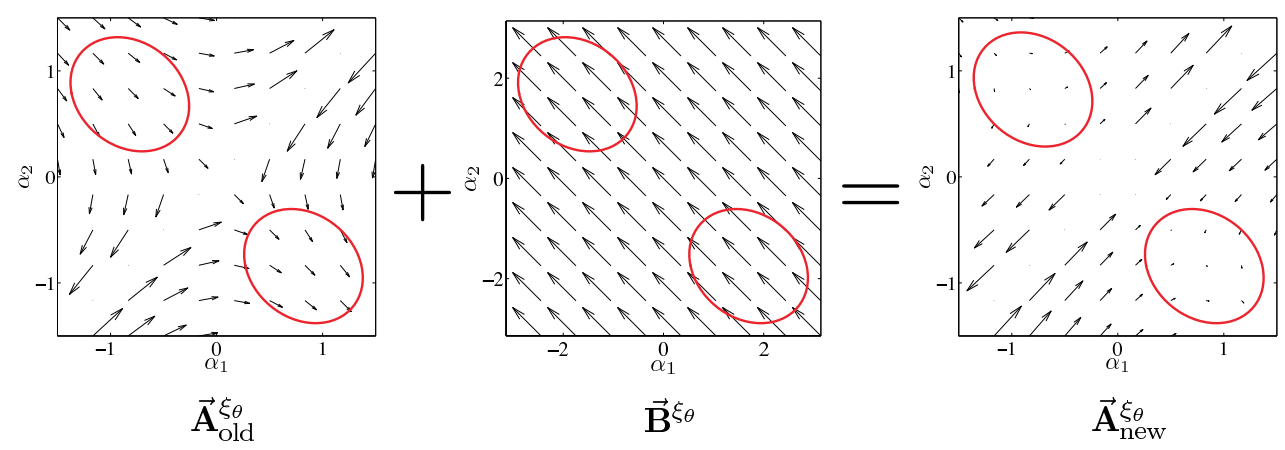

Fig. 9: The effect of adding $\overrightarrow{\mathbf{B}} \xi_{\theta}$ to $\overrightarrow{\mathbf{A}}_{\text {old }}^{\xi_{\theta}}$. In the circled regions, $\overrightarrow{\mathbf{B}}^{\xi_{\theta}}(r) \approx-\overrightarrow{\mathbf{A}}_{\text {old }}^{\xi_{\theta}}(r)$, so $\left\|\overrightarrow{\mathbf{A}}_{\text {new }}^{\xi_{\theta}}\right\| \approx 0$ in these regions, and the BVI will be a good estimate of the displacement resulting from gaits located there. Note that for visual clarity within each plot, the vectors in the different fields are not to scale.
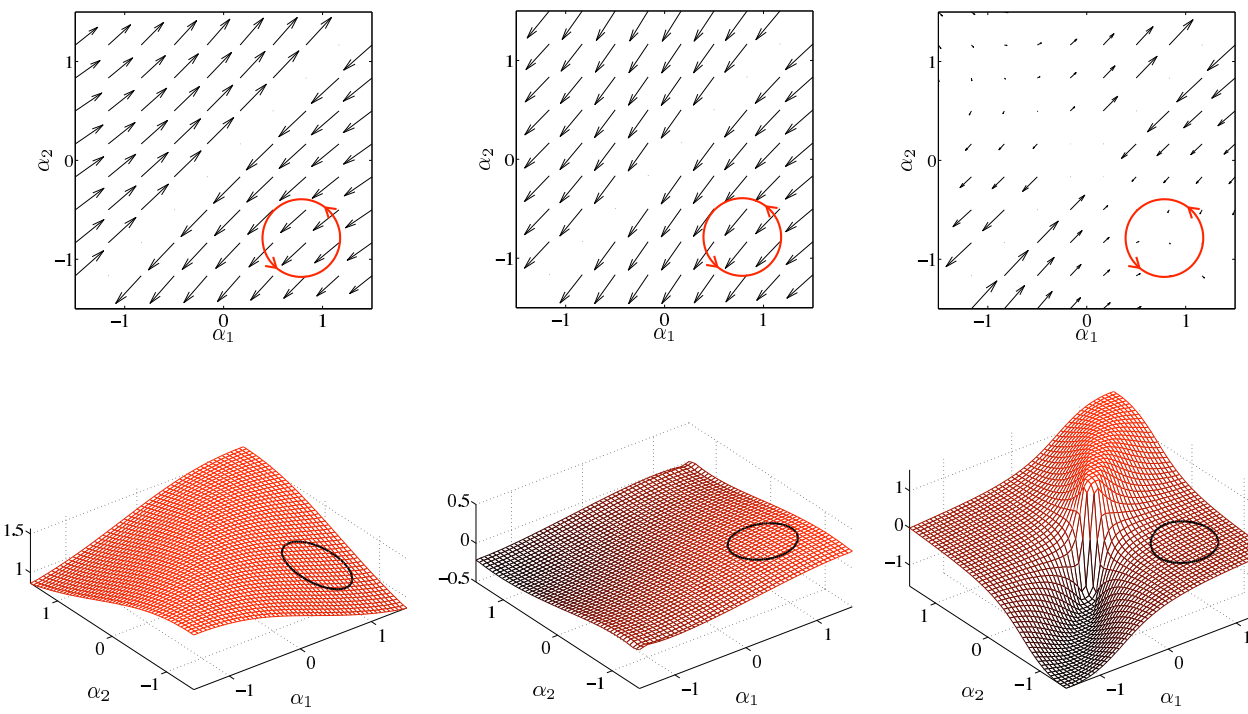

$x$

$y$

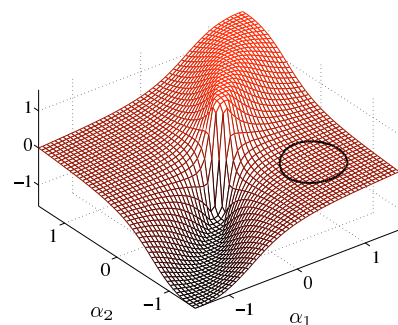

$\theta$

Fig. 10: Example gait image-family with connection vector fields and height functions for kinematic snake with the new mean orientation coordinate choice.

orientation $\theta$ of the system remains close to zero for all time during any of the gaits in the image-family as per (18). From (16), the error term $\varepsilon_{\zeta}$ over the course of these gaits is thus small, and the BVI $\zeta$ is a good approximation of the displacement $g$ resulting from any of the gaits in the imagefamily, as illustrated in Fig. 11.

\section{DEMONSTRATION}

We applied the gaits from the image family in Figs. 5 and 10 to a physical instantiation of the kinematic snake shown in Fig. 12, and plotted the resulting displacements in Fig. 13. During the experiments, we observed some backlash in the joints and slip in the constraints, to which we attribute the differences between the experimental and calculated loci in Figs. 13 and 11. Even with this error, the BVI was a considerably more effective estimate of the displacement with the new coordinate choice than with the old choice. Under the old coordinate choice, the error in the the net direction of travel between the BVI estimate and the actual displacement ranged from 0.72 to 0.25 radians. With the new choice, the error in the estimate ranged from 0.10 to -0.04 radians, reducing the maximum error magnitude by $86 \%$ and eliminating the minimum error.

\section{CONCLUSION}

In this paper, we have shown that the choice of coordinates used to represent a mobile system directly affects the computational cost of evaluating its motion properties. By choosing a set of coordinates which minimizes the rotation of the body frame in response to changes in system shape, we have advanced a technique for approximating the net displacement resulting from a gait by means of Stokes's theorem, rather than by the considerably more expensive integration of the equations of motion. In turn, this approximation provides a means of designing gaits by encircling sign-definite regions of well-defined height functions. The technique constitutes a 


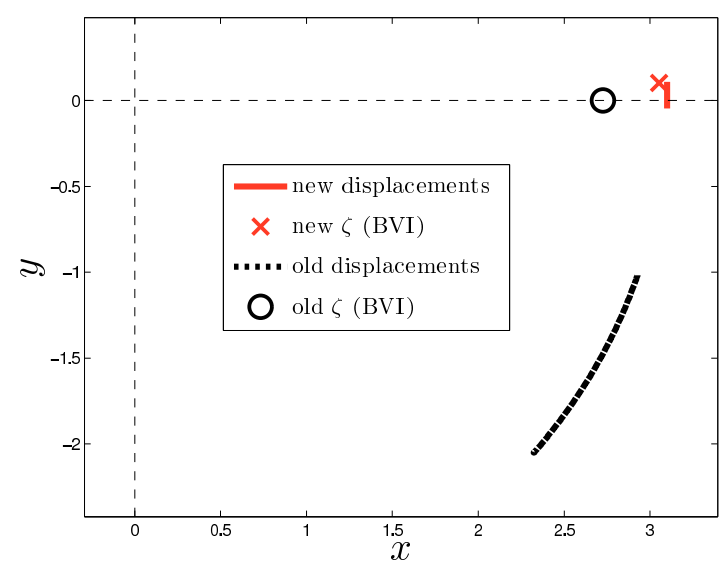

Fig. 11: The BVI and displacements corresponding to the image-family of gaits in Fig. 10 for the kinematic snake robot with the new measure of orientation depicted in Fig. 7 are represented respectively by the cross above the $x$-axis and the short arc-section, with the BVI and displacements as measured in the old coordinate choice presented for reference. With the new measure, the orientation of the system remains almost constant over the course of any of the gaits in the family, and the BVI $\zeta$ is thus a good approximation of the displacements.

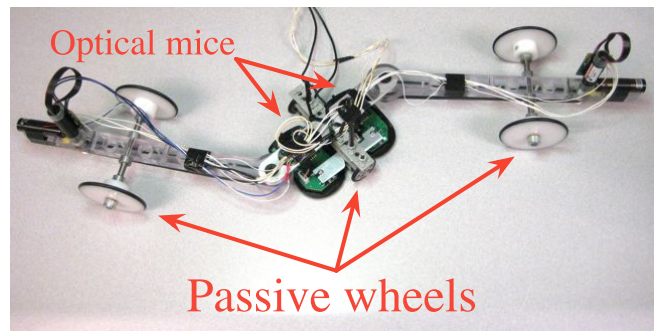

Fig. 12: Physical instantiation of the kinematic snake. Two optical mice on the middle link provide odometry data, including an estimation of constraint slip.

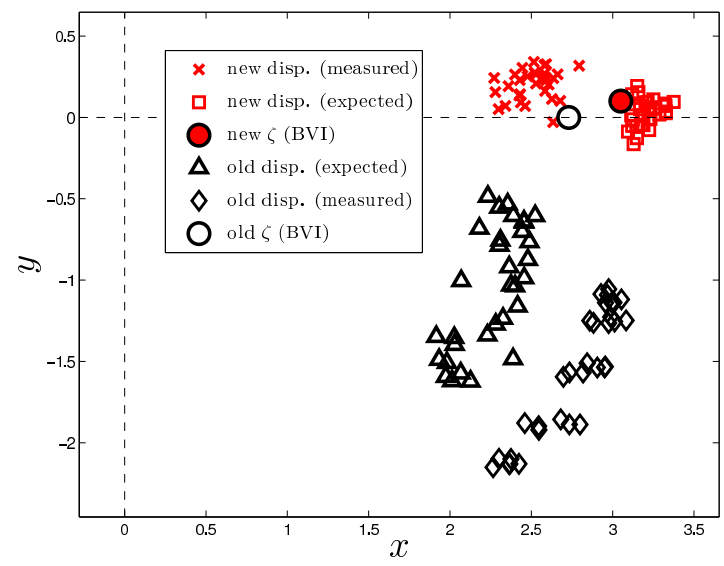

Fig. 13: BVI and displacements for the physical kinematic snake while executing the example image-family of gaits. The boxes show the expected displacements for the robot, calculated from the actual trajectories followed by the joints, while the $\times$ es show the actual measured displacement, which differs from the expected displacement because of backlash and constraint slip. As in Fig. 11, the BVI is a much better estimate of the displacement with the new coordinate choice than with the old choice. significant improvement over previous efforts in this area, as it is applicable to efficient macroscopic translational gaits, while previous work was limited to macroscopic rotational gaits or inefficient small-amplitude translational gaits.

While the coordinate choice presented here, which takes the mean orientation of the links as the orientation of the threelink system, effectively minimizes the body frame rotation, we do not believe it to be the optimal choice. In our future work, we will explore metrics for this minimization, and methods for selecting a coordinate choice which optimizes it for arbitrary systems. Additionally, we will seek to extend the benefits of coordinate choice from the kinematic systems described here to the mixed kinodynamic systems described in [17].

\section{REFERENCES}

[1] A. Shapere and F. Wilczek, "Geometry of self-propulsion at low reynolds number," Geometric Phases in Physics, Jan 1989.

[2] R. Murray and S. Sastry, "Nonholonomic motion planning: steering using sinusoids," IEEE Transactions on Automatic Control, Jan 1993. [Online]. Available: http://eavr.u-strasbg.fr/ bernard/education/ ensps_3a/tmp/murray.pdf

[3] S. Kelly and R. M. Murray, "Geometric phases and robotic locomotion," J. Robotic Systems, Jan 1995. [Online]. Available: ftp://avalon.caltech.edu/pub/murray/preprints/cds/cds94-014.ps.gz

[4] A. M. Bloch et al., Nonholonomic Mechanics and Control. Springer, 2003.

[5] J. Ostrowski and J. Burdick, "The Mechanics and Control of Undulatory Locomotion," International Journal of Robotics Research, vol. 17, no. 7, pp. 683 - 701, July 1998.

[6] E. A. Shammas, H. Choset, and A. A. Rizzi, "Geometric Motion Planning Analysis for Two Classes of Underactuated Mechanical Systems," The International Journal of Robotics Research, vol. 26, no. 10, pp. 1043-1073, 2007. [Online]. Available: http://ijr.sagepub. com/cgi/content/abstract/26/10/1043

[7] J. Ostrowski, J. Desai, and V. Kumar, "Optimal Gait Selection for Nonholonomic Locomotion Systems," International Journal of Robotics Research, 2000.

[8] F. Bullo and K. M. Lynch, "Kinematic controllability for decoupled trajectory planning in underactuated mechanical systems," IEEE Transactions on Robotics and Automation, vol. 17, no. 4, pp. 402-412, August 2001.

[9] K. McIsaac and J. P. Ostrowski, "Motion planning for anguilliform locomotion," Robotics and Automation, Jan 2003. [Online]. Available: http://ieeexplore.ieee.org/xpls/abs_all.jsp?arnumber=1220714

[10] K. Morgansen, B. Triplett, and D. Klein, "Geometric methods for modeling and control of free-swimming fin-actuated underwater vehicles," Robotics, Jan 2007. [Online]. Available: http://ieeexplore. ieee.org/xpls/abs_all.jsp?arnumber=4399955

[11] R. L. Hatton and H. Choset, "Connection vector fields for underactuated systems," IEEE BioRob, October 2008.

[12] R. Mukherjee and D. Anderson, "Nonholonomic Motion Planning Using Stokes' Theorem," in IEEE International Conference on Robotics and Automation, 1993.

[13] G. Walsh and S. Sastry, "On reorienting linked rigid bodies using internal motions," Robotics and Automation, IEEE Transactions on, vol. 11, no. 1, pp. 139-146, January 1995.

[14] E. Shammas, K. Schmidt, and H. Choset, "Natural Gait Generation Techniques for Multi-bodied Isolated Mechanical Systems," in IEEE International Conference on Robotics and Automation, 2005.

[15] J. B. Melli, C. W. Rowley, and D. S. Rufat, "Motion Planning for an Articulated Body in a Perfect Planar Fluid," SIAM Journal of Applied Dynamical Systems, vol. 5, no. 4, pp. 650-669, November 2006.

[16] J. Avron and O. Raz, "A geometric theory of swimming: Purcell's swimmer and its symmetrized cousin," New Journal of Physics, 2008.

[17] E. A. Shammas, H. Choset, and A. A. Rizzi, "Towards a Unified Approach to Motion Planning for Dynamic Underactuated Mechanical Systems with Non-holonomic Constraints," The International Journal of Robotics Research, vol. 26, no. 10, pp. 1075-1124, 2007. [Online]. Available: http://ijr.sagepub.com/cgi/content/abstract/26/10/1075 